\title{
Exome sequencing study revealed novel susceptibility loci in subarachnoid hemorrhage (SAH)
}

\author{
Xiwa Hao ${ }^{\dagger}$, Jiangxia Pang ${ }^{\dagger}$, Ruiming Li, Lin Lv, Guorong Liu, Yuechun Li, Guojuan Cheng and Jingfen Zhang*
}

\begin{abstract}
Aim: To expand our current understanding of the genetic basis of subarachnoid hemorrhage (SAH), and reveal the susceptibility genes in SAH risk.

Methods: We conducted whole-exome sequencing (WES) in a cohort of 196 individuals, including 94 SAH patients and 94 controls, as well as 8 samples that belong to two pedigrees. Systematically examination for rare variations (through direct genotyping) and common variations (through genotyping and imputation) for SAHs were performed in this study.

Results: A total of 16,029 single-nucleotide polymorphisms (SNPs) and 108,999 short indels were detected in all samples, and among them, 30 SNPs distributed on 17 genes presented a strong association signal with SAH. Two novel pathogenic gene variants were identified as associated risk loci, including mutation in TPO and PALD1. The statistical analysis for rare, damaging variations in SAHs identified several susceptibility genes which were involved in degradation of the extracellular matrix and transcription factor signal pathways. And 25 putative pathogenic genes for SAH were also identified basic on functional interaction network analysis with the published SAHassociated genes. Additionally, pedigree analysis revealed autosomal dominant inheritance of pathogenic genes.

Conclusion: Systematical analysis revealed a key role for rare variations in SAH risk and discovered SNPs in new complex loci. Our study expanded the list of candidate genes associated with SAH risk, and will facilitate the investigation of disease-related mechanisms and potential clinical therapies.
\end{abstract}

Keywords: Subarachnoid hemorrhage (SAH), Whole-exome sequencing (WES), Genome-wide association analysis (GWAS), Rare variations, Pedigree analysis

\section{Introduction}

Subarachnoid hemorrhage (SAH), the rarest but most fatal type of stroke, has shown an annual incidence of $8-10 / 100,000$ persons (2007), 30-day case fatality of $35-45 \%$ in western countries $[17,20]$. In China, annual incidence (per 100,000 persons) of SAH was 6.2, which is slightly lower than in western countries [40]. The majority of patients with SAH usually suffered from

\footnotetext{
* Correspondence: 1846224761@qq.com

+Xiwa Hao and Jiangxia Pang contributed equally to this work.

Department of Neurology, Baotou Central Hospital, Baotou, China
}

ruptured intracranial aneurysm (IA). SAH risk was considered to be related to smoking, hypertension, and poor socioeconomic status [2, 14]. Moreover, studies based on molecular mechanisms have shown that genetic factors also play an important role in the formation, growth and rupture of IA $[6,11,24,32,38]$. Therefore, IA is identified as a complex disease that influenced by various genes and environmental factors. Conducting early detection and intervention by identifying risk factors may facilitate to avoid the formation and rupture of IA, and is crucial for the reduced

C C The Author(s). 2020 Open Access This article is licensed under a Creative Commons Attribution 4.0 International License, which permits use, sharing, adaptation, distribution and reproduction in any medium or format, as long as you give appropriate credit to the original author(s) and the source, provide a link to the Creative Commons licence, and indicate if changes were made. The images or other third party material in this article are included in the article's Creative Commons licence, unless indicated otherwise in a credit line to the material. If material is not included in the article's Creative Commons licence and your intended use is not permitted by statutory regulation or exceeds the permitted use, you will need to obtain permission directly from the copyright holder. To view a copy of this licence, visit http://creativecommons.org/licenses/by/4.0/ The Creative Commons Public Domain Dedication waiver (http://creativecommons.org/publicdomain/zero/1.0/) applies to the data made available in this article, unless otherwise stated in a credit line to the data. 
incidence of SAH [12]. However, comprehensive knowledge of pathogenic and ruptured mechanisms of IA has not yet been defined.

Some studies have focused on the pathogenic mechanisms of IA in China, and several susceptibility genes have been identified. Due to the limitation of technology and small sample size, it is still necessary to further study the genetic factors of IA in China. Although genome-wide association analysis (GWAS) studies have found some novel gene loci related to IAs, they can only explain part of the genetic risk. Most of the GWAS studies have focused on both unruptured and ruptured IAs, thus, the gene loci highly related to ruptured IAs have not been completely detected. Moreover, rare, damaging variants also play an important role in complex diseases. With advances in sequencing technology, genetic analysis is gradually extending to rare variants, which often have more obvious functional consequences of harmful phenotypes [13, 29].

In this study, we set out to systematically examine rare variation (through direct genotyping) and common variation (through genotyping and imputation) for SAHs by whole-exome sequencing (WES) in a cohort of 196 samples. Mendelian inheritance analysis for the SAH pedigrees was also performed to identified the susceptibility genes. Our study constitutes a detailed simultaneous assessment of causal variations in a large sample of SAHs, offer an opportunity to better understand both the biological and genetic architecture of this type of complex disease.

\section{Materials and methods}

\section{Study cohorts}

We prospectively collected 196 samples, including 8 samples that belong to two pedigrees from Central Hospital of Baotou. The cohorts included 94 SAH cases(with ruptured intracranial aneurysm confirmed by Digital Subtraction Angiography, DSA and computed tomography, CT) and 94 controls(for each case, 1 control without SAH will be sort for interview. Controls will be matched on the basis of the following criteria:

- gender (sex)

- 10-year age strata (ie 10-19, 20-29, etc)

- sector of suburb of residence in Baotou (North, East, etc).

Controls will be chosen from the spouse, relative or friend of patients without SAH who are currently in the same hospital as the case.). This study was approved by the Human Research Ethics Committee of Central Hospital of Baotou, and all participants provided written informed consent. Comprehensive clinical information was provided in Table S1, including height, weight, BMI, gender and age etc.

\section{Whole-exome sequencing}

Genomic DNA was isolated from peripheral whole blood samples of participants by using Genomic DNA Extraction Kit (Invitrogen, South San Francisco, CA, USA). The Qubit 3.0 fluorometer and gel electrophoresis were used to evaluate DNA quantity and integrity, respectively. The sequencing paired-end libraries were constructed for each sample and captured using SureSelect Human All Exon V6 kit (Agilent Technologies, Santa Clara, CA, USA) following the manufacturer's instructions. All libraries were sequenced on BGI-SEQ 500 platform at BGI to obtain a desired depth of $\sim 100 \mathrm{X}$. The sequencing depths of each sample are listed in Table S2.

\section{Whole-exome sequencing (WES) data processing and variant calling}

To get high quality data, Trimmomatic [5] was used to filter out low-quality reads which contained adaptors, high base error rate $(>50 \%)$, and highly unknown base proportion $(>10 \%)$ from the raw sequencing data. The cleaned reads were aligned to human reference genome (UCSC hg19) by the Burrows-Wheeler Aligner-MEM (v.0.7.15) [26] with default parameters. All the aligned reads were further processed using Picard tools (v2.5.0) and Genome Analysis Toolkit (GATK, v3.7) [28] with default parameters, which included deduplication, base quality recalibration, and multiple-sequence realignment prior to mutation detection.

Variant calling was performed for all the samples by using the Haplotype Caller algorithm in GATK with the parameters “-stand_call_conf 30 -stand_emit_conf 10 -minPruning 3". Each variant was filtered using GATK hard filters with the parameters "QD $<2.0 \quad|| F S>60$ \| $\mathrm{MQ}<40$ || MQRankSum<-12.5 || ReadPosRankSum<8.0" for SNPs and "QD $<2.0 \|$ FS $>200 \|$ ReadPosRankSum < -20" for Indels to reduce the false positive rate. We then called genotypes jointly across all samples at the remaining sites, followed by genotype refinement using the BEAGLE imputation software (v5.0) [7]. The variants were subsequently annotated by multiple databases using the ANNOVAR tool [37].

\section{Sample quality control}

The standard quality screening conducted independently in each sample included SNP and sample call rates (>90\%), Hardy-Weinberg equilibrium, Mendelian errors, gender inconsistencies and checks for population stratification. To obtain a high-quality set of samples, the outlier samples discovered using principal-component analysis in GCTA [39] were removed from further analysis. 


\section{Association testing}

The single marker association analyses with SAH were performed using an additive genetic model implemented in SNPTEST (http://www.stats.ox.ac.uk/ marchini/software/gwas/snptest.html) for the common SNPs (MAF > $10 \%)$. Age, sex, BMI, smoking, drinking, body fat, and diabetes were used as covariates in the analysis.

\section{Rare SNP filtering}

We used different allele frequency threshold in several public population databases: 1000G (http://browser.1 000genomes.org/index.html), ExAC, ESP etc., to filter out common variants. Then, only variants with frequency less than the thresholds in all these databases were considered as the rare SNPs of SAHs.

\section{Functional impact prediction}

Each variant category has to be assessed with a specific set of tools to predict their functional impact. Here, we assumed that synonymous variants have no functional impact, and all the stop gain and stop loss variants were considered as the deleterious mutations. The functional predictions of missense variants were performed by seven computational methods (SIFT (Ng, 2001 \#4097), Polyphen2 [1], MutationTaster [33], CADD [27], REVEL [21], M-CAP [22], LRT [10]). The pathogenicity of missense mutations was assumed if predicted pathogenic by at least five out of the computational methods. The dpsi score were employed to determine the pathogenicity of splicing mutations.

\section{Gene-based burden analysis}

Gene-base test were performed for the rare, damaging variants. For each gene, we computed the burden of rare, damaging variants in SAH cases and controls, respectively. Fisher's exact test was applied to determine the significantly associated genes in SAHs. Those genes with a $P$-value of less than 0.05 were identified as susceptibility genes in SAHs. SKAT-O [25] was also applied for burden test, which allowing for variants with opposite directions of effect to reside in the same gene.

\section{Inheritance analysis in pedigrees}

The SNPs were called from the 2 pedigrees, and were further filtered as the filtering criterion of rare SNPs. Then, all the SNPs were subjected to functional impact prediction. Mendelian inheritance analysis was performed for the diseasing causing SNPs with 4 inheritance patterns, including (1) dominant inheritance pattern; (2) recessive inheritance pattern; (3) semidominant inheritance pattern; (4) compound heterozygote inheritance pattern.

\section{The network analysis}

The SAH-associated genes were collected from the published studies. The STRING database and associated search tools [35] were used for identifying interacting partners of a list of SAH-associated genes. We employed the identified interacting partners as the candidate pathogenic genes in SAHs.

\section{Results}

\section{Cohorts description and whole-exome sequencing}

In this study, we performed $\sim 100 x$ whole-exome sequencing (WES) for 94 SAH cases, 94 controls, and 2 pedigrees. Comprehensive description of the height, weight, sex, age and the other clinical variables of the cohort are provided in Table S1. In brief, the SAH group included 55 hypertension, 10 diabetic, 11 hyperlipidemia, 46 smoker/former-smoker, and 22 drinker/formerdrinker. The control group included 37 hypertension, 9 diabetic, 9 hyperlipidemia, 42 smoker/former-smoker, and 23 drinker/former-drinker. The statistics of the WES data was provided in Table S2 and S3, including effective bases, SNPs numbers, Indel numbers and $\mathrm{Ti} / \mathrm{Tv}$ rate etc. for each sample.

We totally discovered 716,029 single-nucleotide polymorphisms (SNPs) and 108,999 short indels in all the samples. We then applied Genome Analysis Toolkit (GATK) VQSR for SNVs to distinguish true sites of genetic variation from sequencing artifacts. Then, 549,553 SNPs were remained, including 148,967 exonic SNPs, for the further analysis (See Method section, Table S4). Following sample quality control, the whole-exome sequences of 93 patients with SAH and 92 controls were jointly analyzed (See Method section).

\section{Imputation into GWAS}

For imputation purposes, we conducted a genome-wide single-variant analysis of the common SNPs (minor allele frequency, MAF > 0.1) comparing the $93 \mathrm{SAH}$ cases and 92 controls. The associations with SAH risk were tested using logistic regression adjusted for sex, BMI, smoking, drinking, body fat, and diabetes as covariates. The genomic inflation factor $(\lambda=1.006)$ showed no evidence of inflated test statistics. There were 30 SNPs distributed on 17 genes presented a strong association signal with SAH (Table 1, Fig. 1). In these SNPs, three of them were in exon, and one in UTR3, and the other in intron. We obtained two loci reached genome-wide significance, within the introns of two genes TPO and $P A L D 1$, respectively (Fig. 2), implies a putative functional role in the pathogenesis of SAHs.

$T P O$ encodes a membrane-bound glycoprotein that plays a major role in thyroid gland function. Mutations in this gene are associated with several disorders of thyroid hormonogenesis, i.e., congenital hypothyroidism, 
Table 1 SNPs with the strongest association with SAH from the GWAS results

\begin{tabular}{|c|c|c|c|c|c|c|c|c|c|}
\hline Chr & Pos & Ref & Alt & Cases MAF & Controls MAF & OR & $\boldsymbol{P}$ value & Function & Gene \\
\hline 10 & $72,300,743$ & G & C & 0.087 & 0.274 & 3.97 & $6.94 \mathrm{E}-07$ & intronic & PALD1 \\
\hline 2 & $1,437,410$ & $C$ & T & 0.245 & 0.484 & 2.90 & 1.16E-06 & intronic & TPO \\
\hline 2 & $31,189,236$ & A & G & 0.212 & 0.425 & 2.75 & 2.37E-06 & intronic & GALNT14 \\
\hline 2 & $31,189,304$ & $C$ & T & 0.212 & 0.425 & 2.75 & 2.37E-06 & intronic & GALNT14 \\
\hline 2 & $31,189,345$ & $\mathrm{~T}$ & G & 0.212 & 0.425 & 2.75 & 2.37E-06 & intronic & GALNT14 \\
\hline 2 & $31,189,401$ & G & A & 0.212 & 0.425 & 2.75 & 2.37E-06 & intronic & GALNT14 \\
\hline 2 & $31,189,439$ & A & G & 0.212 & 0.425 & 2.75 & 2.37E-06 & intronic & GALNT14 \\
\hline 10 & $72,306,967$ & T & $C$ & 0.174 & 0.366 & 2.74 & 3.33E-06 & intronic & PALD1 \\
\hline 10 & $72,306,978$ & A & C & 0.174 & 0.366 & 2.74 & 3.33E-06 & intronic & PALD1 \\
\hline 2 & $1,437,163$ & $C$ & A & 0.168 & 0.376 & 2.98 & $6.23 \mathrm{E}-06$ & intronic & TPO \\
\hline 19 & $50,189,818$ & C & G & 0.098 & 0.016 & 0.15 & 1.09E-05 & intronic & PRMT1 \\
\hline 20 & $3,846,843$ & T & $C$ & 0.348 & 0.172 & 0.39 & 1.34E-05 & UTR3 & MAVS \\
\hline 10 & $72,289,778$ & T & $C$ & 0.109 & 0.269 & 3.01 & 1.82E-05 & exonic & PALD1 \\
\hline 19 & $50,195,455$ & $A$ & G & 0.092 & 0.016 & 0.16 & 2.38E-05 & intronic & CPT1C \\
\hline 4 & $1.85 \mathrm{E}+08$ & A & G & 0.147 & 0.032 & 0.19 & 2.65E-05 & intronic & TRAPPC11 \\
\hline 7 & $28,449,965$ & $C$ & T & 0.033 & 0.140 & 4.82 & 2.73E-05 & intronic & CREB5 \\
\hline 2 & $1,442,417$ & T & C & 0.163 & 0.355 & 2.82 & 2.80E-05 & intronic & TPO \\
\hline 2 & $1,442,476$ & $C$ & $\mathrm{~T}$ & 0.163 & 0.355 & 2.82 & 2.80E-05 & intronic & TPO \\
\hline 5 & $58,334,645$ & G & A & 0.054 & 0.199 & 4.32 & 3.43E-05 & intronic & PDE4D \\
\hline 10 & $72,307,101$ & $C$ & T & 0.603 & 0.398 & 2.30 & $3.58 \mathrm{E}-05$ & exonic & PALD1 \\
\hline 14 & $35,062,166$ & $\mathrm{~T}$ & $C$ & 0.114 & 0.263 & 2.78 & $4.50 \mathrm{E}-05$ & intronic & SNX6 \\
\hline 7 & $1.51 \mathrm{E}+08$ & G & $C$ & 0.315 & 0.516 & 2.32 & 4.78E-05 & intronic & NUB1 \\
\hline 18 & $14,796,080$ & A & G & 0.234 & 0.382 & 2.02 & 5.39E-05 & intronic & ANKRD3OB \\
\hline 2 & $1,426,621$ & A & G & 0.332 & 0.532 & 2.29 & $6.31 \mathrm{E}-05$ & intronic & TPO \\
\hline 9 & $1.02 \mathrm{E}+08$ & T & $C$ & 0.196 & 0.065 & 0.28 & $6.60 \mathrm{E}-05$ & intronic & GALNT12 \\
\hline 15 & $23,049,369$ & A & G & 0.337 & 0.177 & 0.42 & 8.36E-05 & intronic & NIPA1 \\
\hline 5 & $75,427,935$ & A & G & 0.272 & 0.452 & 2.21 & 9.09E-05 & exonic & $S V 2 C$ \\
\hline 10 & $72,288,900$ & G & A & 0.397 & 0.591 & 2.20 & 0.000103 & intronic & PALD1 \\
\hline 10 & $50,683,438$ & $C$ & T & 0.277 & 0.118 & 0.35 & 0.000104 & intronic & ERCC6 \\
\hline 6 & $70,970,299$ & $\mathrm{~T}$ & C & 0.223 & 0.091 & 0.35 & 0.000106 & intronic & COL9A1 \\
\hline
\end{tabular}

and congenital goiter [31,34]. As depicted in Fig. 2, another SNP within Phosphatase Domain Containing Paladin 1 (PALD1) also showed a significant signal. PALD1 is thought to be involved in the formation of vascular endothelium [36].

\section{The role of rare variations in SAH risk}

It is plausible that analysis of rare variants could explain additional disease risk or trait variability. We next investigated the rare variants across the cohorts by applying the frequency filtering (see Method section). The variants were defined as rare if their frequency in various databases were less than the corresponding threshold (Table S5). Each rare variant was assessed with a specific set of tools to predict their functional impact (see Method section).
The 30,651 potential damaging rare variants remained for further analysis.

We employed two gene-based methods to identify the susceptibility genes in SAHs. Rare variants burden testing was performed between SAH cases and control samples by Fisher's exact test, and 38 susceptibility genes, such as gene OBSCN, TJP1, ADGRV1, and FBN3 etc., were obtained (Table 2). However, when variants with opposite directions of effect in the same gene, the testing power will be reduced. We then employed another analysis with SKAT-O [25] to identify the signals, which both allowed for variants with opposite directions of effect to reside in the same gene. The SKAT-O identified 37 signals (Table 3), which were highly overlap with the results from the burden test (92.3\%, Fig. 3), which suggested that these genes could be directly involved in ALS risk. 


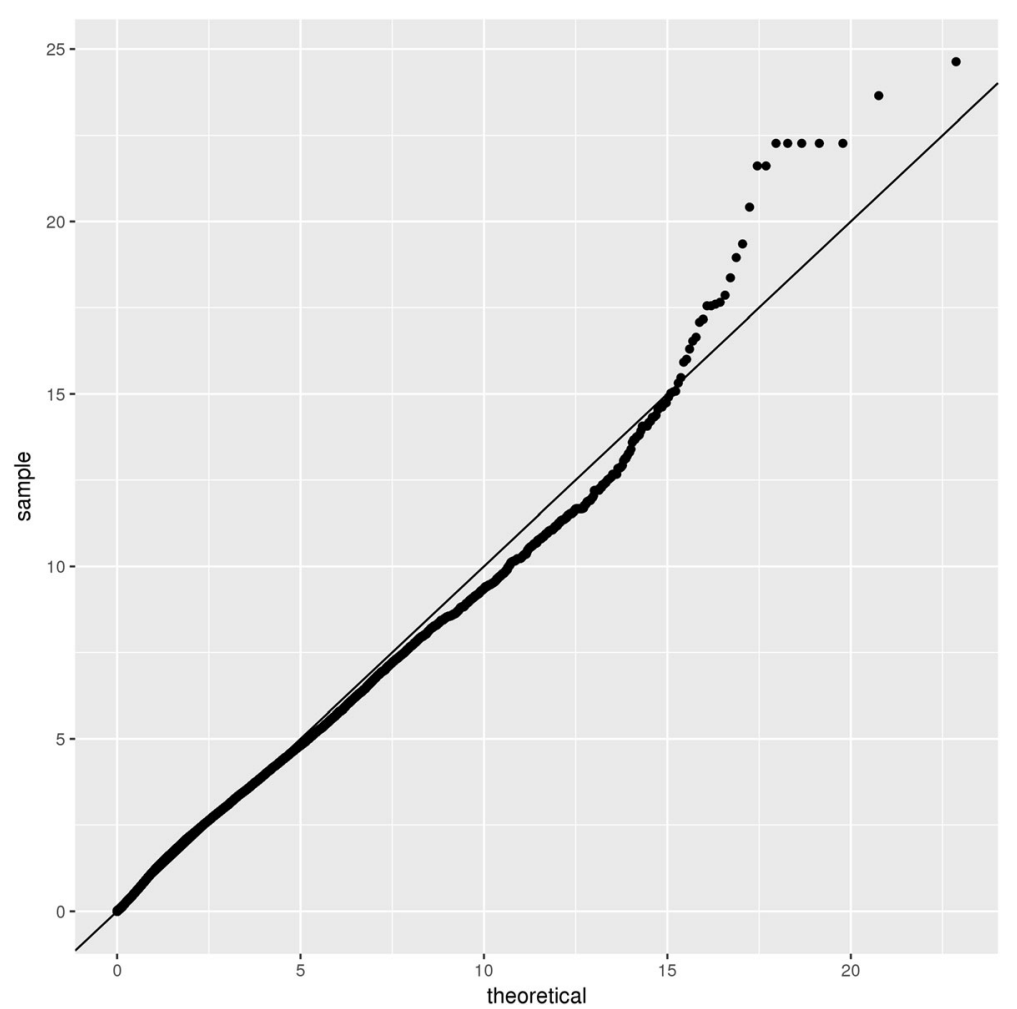

Fig. 1 Quantile-quantile (Q-Q) plots of the meta-analyses of genome-wide association studies (GWAS) results for SAH

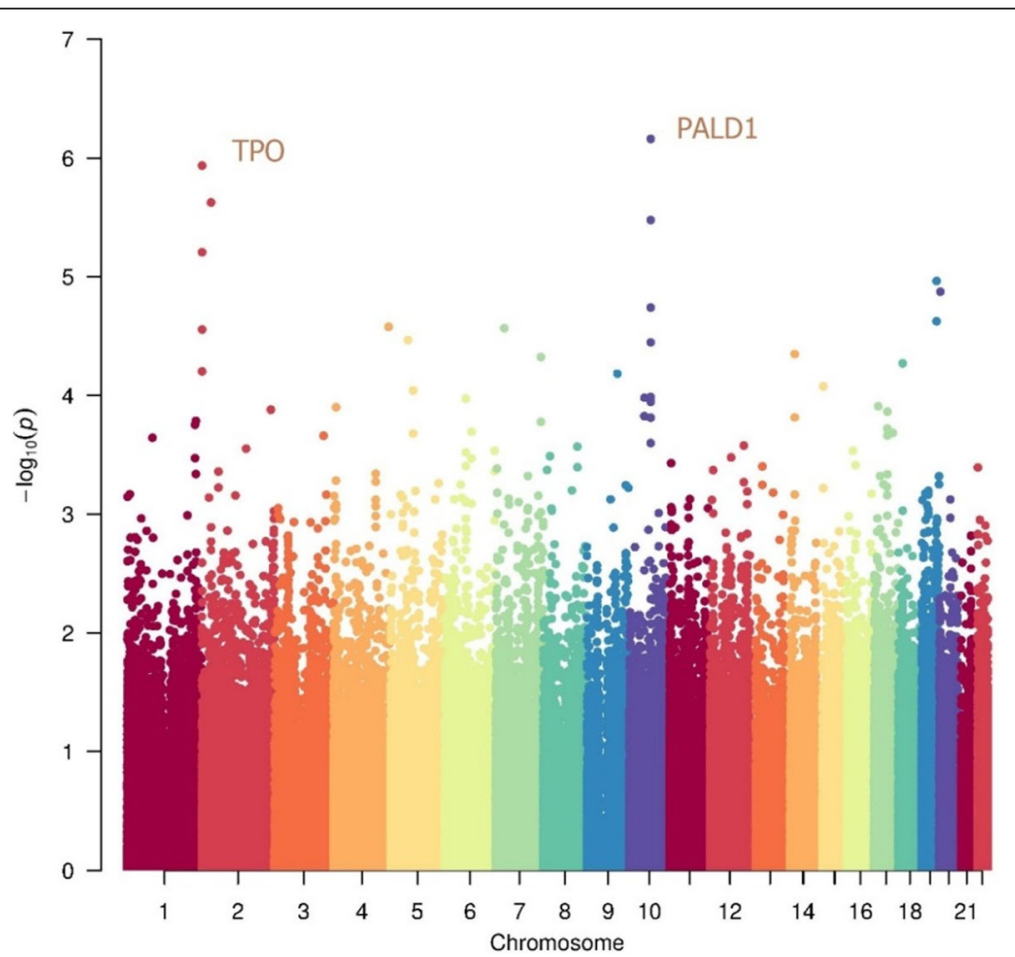

Fig. 2 Manhattan plot depicting the GWAS results for SAH. Each dot represents a single-nucleotide polymorphism (SNP), with the chromosomal position on the $\mathrm{x}$ axis and the $P$-value on the $\mathrm{y}$ axis 
Table 2 Candidate genes in SAH identified by burden test of rare variants

\begin{tabular}{|c|c|c|c|c|c|c|}
\hline Gene & $\begin{array}{l}\text { Number of Cases } \\
\text { with mutations }\end{array}$ & $\begin{array}{l}\text { Number of Controls } \\
\text { with mutations }\end{array}$ & $\begin{array}{l}\text { Number of Cases } \\
\text { without mutations }\end{array}$ & $\begin{array}{l}\text { Number of Controls } \\
\text { without mutations }\end{array}$ & $P$ value & OR \\
\hline OBSCN & 26 & 11 & 68 & 82 & 0.005 & 2.834 \\
\hline$A B C G 8$ & 7 & 0 & 87 & 93 & 0.007 & $\operatorname{lnf}$ \\
\hline PIGG & 7 & 0 & 87 & 93 & 0.007 & $\operatorname{lnf}$ \\
\hline GOLGA2 & 6 & 0 & 88 & 93 & 0.015 & $\operatorname{lnf}$ \\
\hline MTMR4 & 6 & 0 & 88 & 93 & 0.015 & $\operatorname{lnf}$ \\
\hline MYHI & 6 & 0 & 88 & 93 & 0.015 & $\operatorname{lnf}$ \\
\hline OTOGL & 6 & 0 & 88 & 93 & 0.015 & $\operatorname{lnf}$ \\
\hline TCF3 & 6 & 0 & 88 & 93 & 0.015 & $\operatorname{lnf}$ \\
\hline TJP1 & 10 & 2 & 84 & 91 & 0.017 & 5.374 \\
\hline KMT2C & 8 & 1 & 86 & 92 & 0.018 & 8.482 \\
\hline ADGRV1 & 16 & 6 & 78 & 87 & 0.021 & 2.958 \\
\hline FBN3 & 9 & 2 & 85 & 91 & 0.030 & 4.782 \\
\hline$A B C G 5$ & 5 & 0 & 89 & 93 & 0.030 & $\operatorname{lnf}$ \\
\hline BCL9 & 5 & 0 & 89 & 93 & 0.030 & $\operatorname{lnf}$ \\
\hline Clorf94 & 5 & 0 & 89 & 93 & 0.030 & $\operatorname{lnf}$ \\
\hline CEBPZ & 5 & 0 & 89 & 93 & 0.030 & $\operatorname{lnf}$ \\
\hline COG3 & 5 & 0 & 89 & 93 & 0.030 & $\operatorname{lnf}$ \\
\hline CTC1 & 5 & 0 & 89 & 93 & 0.030 & $\operatorname{lnf}$ \\
\hline FDXR & 5 & 0 & 89 & 93 & 0.030 & $\operatorname{lnf}$ \\
\hline GRIK3 & 5 & 0 & 89 & 93 & 0.030 & Inf \\
\hline INCENP & 5 & 0 & 89 & 93 & 0.030 & $\operatorname{lnf}$ \\
\hline IQGAP3 & 5 & 0 & 89 & 93 & 0.030 & $\operatorname{lnf}$ \\
\hline KNDC1 & 5 & 0 & 89 & 93 & 0.030 & $\operatorname{lnf}$ \\
\hline LETMD1 & 5 & 0 & 89 & 93 & 0.030 & $\operatorname{lnf}$ \\
\hline METTL22 & 5 & 0 & 89 & 93 & 0.030 & $\operatorname{lnf}$ \\
\hline NCOA6 & 5 & 0 & 89 & 93 & 0.030 & $\operatorname{lnf}$ \\
\hline PDZD7 & 5 & 0 & 89 & 93 & 0.030 & $\operatorname{lnf}$ \\
\hline PIF1 & 5 & 0 & 89 & 93 & 0.030 & $\operatorname{lnf}$ \\
\hline PLXNA4 & 5 & 0 & 89 & 93 & 0.030 & $\operatorname{lnf}$ \\
\hline RAPGEFL 1 & 5 & 0 & 89 & 93 & 0.030 & $\operatorname{lnf}$ \\
\hline RGS14 & 5 & 0 & 89 & 93 & 0.030 & $\operatorname{lnf}$ \\
\hline SCN7A & 5 & 0 & 89 & 93 & 0.030 & Inf \\
\hline THEG & 5 & 0 & 89 & 93 & 0.030 & $\operatorname{lnf}$ \\
\hline VEPH1 & 5 & 0 & 89 & 93 & 0.030 & $\operatorname{lnf}$ \\
\hline ZFP9O & 5 & 0 & 89 & 93 & 0.030 & Inf \\
\hline LENG8 & 7 & 1 & 87 & 92 & 0.033 & 7.339 \\
\hline NIPBL & 7 & 1 & 87 & 92 & 0.033 & 7.339 \\
\hline TECPR2 & 7 & 1 & 87 & 92 & 0.033 & 7.339 \\
\hline
\end{tabular}

The overlapped genes were further subjected to functional enrichment analysis. These genes were overrepresented in some pathways related to cellular organization, i.e., adherens junction, and degradation of the extracellular matrix; and transcription factor signal, i.e., TGF-beta signal pathway (Table 4).
Pedigree analysis

We performed Mendelian inheritance analysis for two $\mathrm{SAH}$ pedigrees with probable inheritance patterns, including (1) dominant inheritance pattern; (2) recessive inheritance pattern; (3) semi-dominant inheritance pattern; (4) compound heterozygote inheritance pattern. Pathogenicity of missense mutations was assumed if 
Table 3 Candidate genes in SAH identified by SKAT-O analysis

\begin{tabular}{|c|c|c|c|c|c|c|c|}
\hline Gene & $P$ value & Number of Marker All & Number of Marker Test & MAC & $\mathrm{m}$ & Method bin & MAP \\
\hline$\overline{O B S C N}$ & 0.005 & 37 & 36 & 40 & 37 & ER.A & -1.000 \\
\hline$A B C G 8$ & 0.010 & 8 & 7 & 7 & 7 & ER & 0.003 \\
\hline PIGG & 0.010 & 9 & 7 & 7 & 7 & ER & 0.003 \\
\hline GOLGA2 & 0.021 & 6 & 6 & 6 & 6 & ER & 0.007 \\
\hline MTMR4 & 0.021 & 7 & 7 & 7 & 6 & ER & 0.007 \\
\hline MYH1 & 0.021 & 6 & 6 & 6 & 6 & ER & 0.007 \\
\hline TCF3 & 0.021 & 6 & 6 & 6 & 6 & ER & 0.007 \\
\hline KMT2C & 0.026 & 9 & 9 & 9 & 9 & ER & 0.001 \\
\hline FBN3 & 0.039 & 13 & 12 & 12 & 11 & ER & 0.000 \\
\hline$A B C G 5$ & 0.044 & 5 & 5 & 5 & 5 & ER & 0.014 \\
\hline$B C L 9$ & 0.044 & 5 & 5 & 5 & 5 & ER & 0.014 \\
\hline Clorf94 & 0.044 & 5 & 5 & 5 & 5 & ER & 0.014 \\
\hline CCDC102A & 0.044 & 7 & 5 & 5 & 5 & ER & 0.014 \\
\hline CEBPZ & 0.044 & 5 & 5 & 5 & 5 & ER & 0.014 \\
\hline COG3 & 0.044 & 4 & 4 & 5 & 5 & ER & 0.014 \\
\hline CTC1 & 0.044 & 5 & 5 & 5 & 5 & ER & 0.014 \\
\hline FDXR & 0.044 & 5 & 5 & 5 & 5 & ER & 0.014 \\
\hline GRIK3 & 0.044 & 5 & 5 & 5 & 5 & ER & 0.014 \\
\hline INCENP & 0.044 & 3 & 3 & 5 & 5 & ER & 0.014 \\
\hline IQGAP3 & 0.044 & 6 & 5 & 5 & 5 & ER & 0.014 \\
\hline KNDC1 & 0.044 & 5 & 5 & 5 & 5 & ER & 0.014 \\
\hline LETMD1 & 0.044 & 4 & 4 & 5 & 5 & ER & 0.014 \\
\hline METTL22 & 0.044 & 4 & 4 & 5 & 5 & ER & 0.014 \\
\hline NCOAG & 0.044 & 5 & 5 & 5 & 5 & ER & 0.014 \\
\hline OTOGL & 0.044 & 5 & 4 & 5 & 5 & ER & 0.014 \\
\hline PDZD7 & 0.044 & 3 & 3 & 5 & 5 & ER & 0.014 \\
\hline PIF1 & 0.044 & 6 & 5 & 5 & 5 & ER & 0.014 \\
\hline PLXNA4 & 0.044 & 4 & 4 & 5 & 5 & ER & 0.014 \\
\hline RAPGEFL1 & 0.044 & 3 & 3 & 5 & 5 & ER & 0.014 \\
\hline RGS14 & 0.044 & 4 & 4 & 5 & 5 & ER & 0.014 \\
\hline SCNTA & 0.044 & 5 & 5 & 5 & 5 & ER & 0.014 \\
\hline THEG & 0.044 & 5 & 5 & 5 & 5 & ER & 0.014 \\
\hline VEPHI & 0.044 & 5 & 5 & 5 & 5 & ER & 0.014 \\
\hline ZFP90 & 0.044 & 4 & 4 & 5 & 5 & ER & 0.014 \\
\hline LENG8 & 0.050 & 8 & 8 & 8 & 8 & ER & 0.002 \\
\hline NIPBL & 0.050 & 8 & 8 & 8 & 8 & ER & 0.002 \\
\hline TECPR2 & 0.050 & 9 & 8 & 8 & 8 & ER & 0.002 \\
\hline
\end{tabular}

predicted pathogenic by at least five out of seven computational methods (SIFT, PolyPhen2, LRT, MutatationTaster, M-CAP, CADD, and REVEL). The potential disease causing variants were only performed in dominant inheritance pattern, and there were 35 and 15 SNPs identified in these two pedigrees, respectively (Table 5 and 6). Twelve and seven candidate genes were identified in pedigree 1 and 2, respectively (Table 7). The gene COL1A2, a pathogenic gene in pedigree 2, was also reported to be associated with SAH phenotype [15].

\section{Putative pathogenic genes for SAHs}

Protein-protein interactions were known as mediating many cellular functions, including cell cycle progression, 


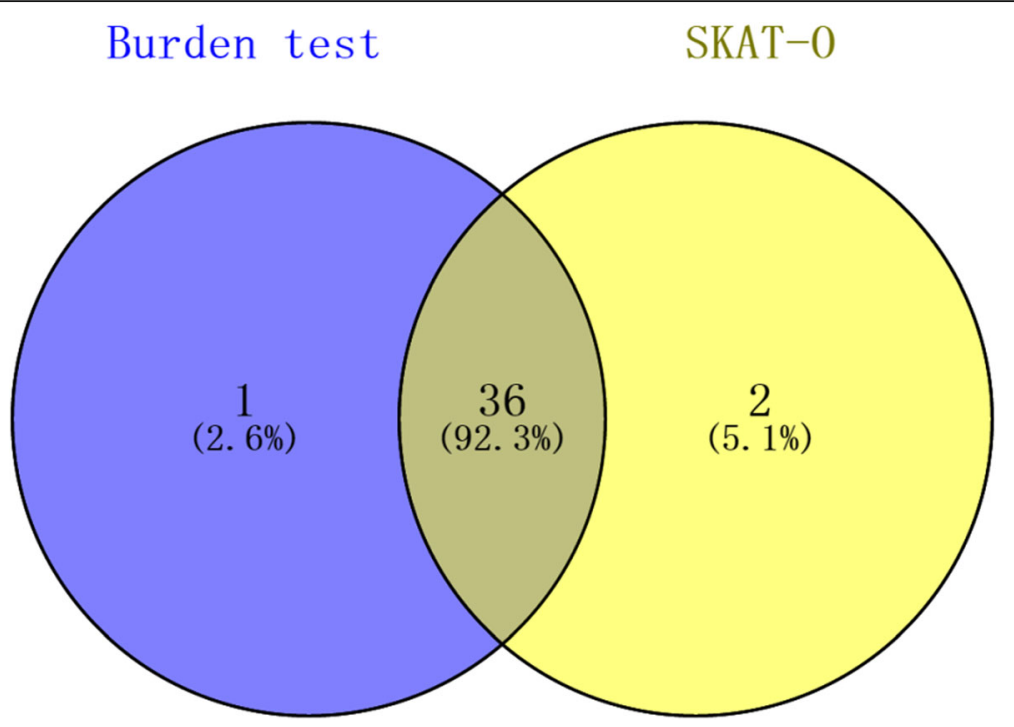

Fig. 3 The number of overlapped genes between burden test and SKAT-O analysis

Table 4 The enriched pathways for the overlapped genes between burden test and SKAT-O analysis

\begin{tabular}{|c|c|}
\hline Pathways & Genes \\
\hline Adherens_junction & TJP1 PTPRM \\
\hline Angiogenesis & FGFR2 UNC5B NOTCH3 FGFR4 FLT4 \\
\hline Apelin_signaling_pathway & NOTCH3 ADCY8 \\
\hline Cell_cycle_Role_of_SCF_complex_in_cell_cycle_regulation & FZR1 MAPK8 \\
\hline \multirow[t]{3}{*}{ Degradation_of_the_extracellular_matrix } & FBN3 ADAMTS18 NCAM1 NTN4 \\
\hline & LAMB2 LAMB1 CAPN1 COL2OA1 FBLN2 \\
\hline & COL14A1 ITGA2 NCAN P4HA2 \\
\hline Development_TGF-beta_receptor_signaling & FZR1 \\
\hline Elastic_fibre_formation & FBN3 FBLN2 \\
\hline Endochondral_Ossification & RUNX2 \\
\hline \multirow[t]{4}{*}{ ERK_signaling } & MYH1 TCF3 FBN3 ECM2 FGFR2 PRKCQ RPS6KA1 FLT4 \\
\hline & TCF19 LAMB2 LAMB1 CAPN1 COL20A1 RASGRP1 \\
\hline & NOTCH3 ADCY8 ARHGEF16 CDH12 CDH19 COL14A1 FGFR4 \\
\hline & IL12RB1 ITGA2 MAPK8 NCAN NTRK3 PLCD4 ARHGEF2 \\
\hline HTLV-I_infection & TCF3 POLE ADCY8 CRTC3 HLA-DPA1 MAPK8 \\
\hline \multirow[t]{2}{*}{ Integrin_Pathway } & MYH1 FBN3 ECM2 PRKCQ LAMB2 LAMB1 CAPN1 \\
\hline & COL20A1 ADCY8 CD36 COL14A1 ITGA2 MAPK8 NCAN \\
\hline \multirow[t]{3}{*}{ PAK_Pathway } & MYH1 TCF3 TJP1 FGFR2 NOX4 PRKCQ PTPRH \\
\hline & TCF19 NOTCH3 FGFR4 FLT4 IL12RB1 \\
\hline & MAPK8 NTRK3 PLCD4 PTPN3 PTPRM GPLD1 \\
\hline Sertoli-Sertoli_Cell_Junction_Dynamics & MYH1 TJP1 SAFB ITGA2 MAPK8 RAB17 RAB34 ARHGEF2 \\
\hline SMAD_Signaling_Network & PSMB8 PSMD5 \\
\hline Smooth_Muscle_Contraction & NULL \\
\hline TGF-beta_receptor_signaling_activates_SMADs & MTMR4 \\
\hline TGF-beta_receptor_signaling & ZFYVE16 \\
\hline TGF-beta_signaling_pathway_KEGG & ID4 INHBA SMAD6 ZFYVE16 \\
\hline TGF-beta_Signaling_Pathways & MAPK8 RUNX2 \\
\hline
\end{tabular}


Table 5 The potential disease causing SNPs in dominant inheritance pattern for pedigree 1

\begin{tabular}{|c|c|c|c|c|c|c|c|c|c|c|}
\hline Chr & Pos & Function & Gene & SIFT & Pp2 & LRT & MT & M-CAP & CADD & REVEL \\
\hline 1 & $2.18 \mathrm{E}+08$ & exonic & GPATCH2 & 0.00 & 1.00 & $D$ & $\mathrm{D}$ & 0.23 & 34.0 & 0.65 \\
\hline 1 & $2.24 \mathrm{E}+08$ & exonic & CCDC185 & 0.04 & 1.00 & & D & 0.02 & 19.6 & 0.35 \\
\hline 1 & $2.25 E+08$ & exonic & DNAHI4 & 0.01 & 0.56 & $U$ & $\mathrm{D}$ & 0.04 & 23.0 & 0.09 \\
\hline 2 & $54,609,069$ & intergenic & C2orf73 SPTBN1 & 0.00 & . & & $N$ & 0.00 & 0.2 & 0.03 \\
\hline 2 & $55,795,456$ & exonic & PPP4R3B & . & 0.39 & $\mathrm{D}$ & $\mathrm{D}$ & 0.03 & 23.6 & 0.72 \\
\hline 2 & $1.79 E+08$ & exonic & $\pi N$ & 0.23 & 0.02 & 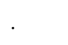 & N & 0.05 & 14.1 & 0.12 \\
\hline 2 & $1.8 \mathrm{E}+08$ & exonic & $\pi N$ & 0.16 & 0.80 & . & $\mathrm{D}$ & 0.03 & 19.0 & 0.44 \\
\hline 2 & $2.2 \mathrm{E}+08$ & exonic & CFAP65 & 0.00 & 0.98 & $N$ & $\mathrm{D}$ & 0.01 & 34.0 & 0.19 \\
\hline 2 & $2.2 \mathrm{E}+08$ & exonic & STK11IP & & & $N$ & A & & 39.0 & \\
\hline 2 & $2.23 E+08$ & exonic & PAX3 & 0.02 & 0.22 & $D$ & $\mathrm{D}$ & 0.07 & 21.4 & 0.48 \\
\hline 3 & $49,169,107$ & exonic & LAMB2 & 0.03 & 0.15 & $\mathrm{~N}$ & D & 0.02 & 22.2 & 0.11 \\
\hline 3 & $1.83 \mathrm{E}+08$ & intronic & ATP11B & 0.03 & . &. & $\mathrm{N}$ & 0.01 & 5.8 & 0.02 \\
\hline 3 & $1.94 \mathrm{E}+08$ & exonic & ATP13A3 & 0.32 & 0.15 & $N$ & $N$ & 0.03 & 6.8 & 0.25 \\
\hline 4 & 871,443 & exonic & GAK & 0.06 & 0.42 & D & $\mathrm{D}$ & 0.07 & 26.3 & 0.53 \\
\hline 4 & $6,873,370$ & exonic & KIAA0232 & 0.04 & 0.20 & $D$ & $D$ & 0.01 & 25.1 & 0.23 \\
\hline 4 & $74,276,089$ & exonic & $A L B$ & 0.03 & 1.00 & $N$ & $\mathrm{~N}$ & 0.05 & 22.4 & 0.02 \\
\hline 7 & $72,397,374$ & exonic & POM121 & 0.07 & 0.51 & $N$ & $N$ & 0.01 & 23.2 & 0.03 \\
\hline 7 & $87,179,859$ & exonic & $A B C B 1$ & 0.18 & 0.01 & D & D & 0.05 & 14.0 & 0.26 \\
\hline 7 & $1.17 \mathrm{E}+08$ & exonic & CTTNBP2 & 0.01 & 0.99 & $D$ & $D$ & 0.04 & 27.4 & 0.36 \\
\hline 7 & $1.29 \mathrm{E}+08$ & exonic & IRF5 & 0.21 & 0.00 & $\mathrm{~N}$ & $N$ & 0.03 & 15.1 & 0.25 \\
\hline 7 & $1.3 \mathrm{E}+08$ & exonic & CPA4 & 0.00 & 0.89 & $D$ & $\mathrm{D}$ & 0.30 & 25.1 & 0.31 \\
\hline 9 & $1.31 E+08$ & exonic & ODF2 & 0.00 & 0.99 & $D$ & $D$ & 0.02 & 28.3 & 0.31 \\
\hline 12 & $6,458,130$ & exonic & SCNN1A & 0.15 & . & & $D$ & 0.10 & 13.7 & 0.09 \\
\hline 14 & $95,562,384$ & exonic & DICER1 & 0.18 & 0.00 & N & $N$ & 0.05 & 0.5 & 0.02 \\
\hline 16 & $89,865,550$ & intronic & FANCA & 0.00 & . & . & $N$ & 0.01 & 3.8 & . \\
\hline 17 & $7,231,013$ & exonic & NEURL4 & 0.00 & 0.01 & $\mathrm{D}$ & $D$ & 0.04 & 22.7 & 0.26 \\
\hline 17 & $7,483,148$ & exonic & CD68 & 0.00 & 0.19 & $\mathrm{~N}$ & D & 0.02 & 22.5 & 0.19 \\
\hline 17 & $7,691,426$ & exonic & DNAH2 & 0.08 & 0.43 & $N$ & $D$ & 0.01 & 22.5 & 0.06 \\
\hline 17 & $73,564,902$ & exonic & LLGL2 & 0.01 & 0.71 & D & $\mathrm{D}$ & 0.04 & 27.4 & 0.53 \\
\hline 17 & $74,085,300$ & exonic & EXOC7 & 0.22 & 0.02 & $\mathrm{D}$ & $D$ & 0.00 & 17.8 & 0.08 \\
\hline 20 & $55,777,539$ & exonic & BMP7 & 0.01 & 0.74 & D & $\mathrm{D}$ & 0.08 & 29.0 & 0.22 \\
\hline 21 & $46,929,308$ & exonic & COL18A1 & 0.14 & 0.16 & $N$ & $N$ & 0.10 & 10.5 & 0.12 \\
\hline 22 & $30,074,259$ & exonic & NF2 & 0.59 & 0.02 & $\mathrm{D}$ & $D$ & 0.08 & 18.4 & 0.43 \\
\hline 22 & $50,721,594$ & exonic & PLXNB2 & 0.51 & 0.00 & $N$ & $N$ & 0.05 & 14.3 & 0.27 \\
\hline 22 & $50,945,311$ & exonic & LMF2 & 0.00 & 0.99 & $D$ & $D$ & 0.67 & 28.0 & 0.41 \\
\hline
\end{tabular}

signal transduction, and metabolic pathways. The genes that interacted with the known SAH genes may influence the SAH phenotypes by participating in the same network/pathway. Basic on previous studies, we collected 28 SAH associated genes (Table S6), and these genes were further assessed the direct and indirect associations with other genes by STRING [35]. In total, we identified 47 putative interacted genes with the SAH (Table S7). To look deep into the pathogenic genes associated with $\mathrm{SAH}$, we selected the overlapped genes among the results from the burden test, SKAT-O analysis and putative interacted genes (Table 8). Finally, we identified 25 putative pathogenic genes for SAH.

Among these genes, FBLN2 has been identified a member of fibulin family, and is responsible for maintenance of the adult vessel wall after injury [8]. BMP7 was reported to play an important role in facilitating recovery after stroke in rat [9]. ITGA2 is responsible for adhesion of platelets and other cells to collagens 
Table 6 The potential disease causing SNPs in dominant inheritance pattern for pedigree 2

\begin{tabular}{|c|c|c|c|c|c|c|c|c|c|c|}
\hline Chr & Pos & Function & Gene & SIFT & Pp2 & LRT & MT & M-CAP & CADD & REVEL \\
\hline 1 & $1.5 \mathrm{E}+08$ & exonic & HIST2H2AC & 0.00 & 0.10 & $\mathrm{~N}$ & $D$ & 0.024 & 8.0 & 0.15 \\
\hline 1 & $1.52 \mathrm{E}+08$ & exonic & RPTN & 0.01 & 0.02 & & N & 0.003 & 16.3 & 0.01 \\
\hline 1 & $1.57 \mathrm{E}+08$ & exonic & IQGAP3 & 0.00 & 1.00 & $D$ & $D$ & 0.154 & 35.0 & 0.84 \\
\hline 1 & $1.62 E+08$ & exonic & DUSP12 & 0.10 & 1.00 & $D$ & $D$ & 0.009 & 23.4 & 0.16 \\
\hline 6 & $56,471,328$ & intronic & DST & 0.01 & 0.17 & $N$ & . & 0.033 & 12.0 & 0.10 \\
\hline 7 & $20,782,555$ & exonic & $A B C B 5$ & 0.00 & 0.99 & $D$ & $D$ & 0.039 & 27.8 & 0.70 \\
\hline 7 & $29,132,261$ & exonic & $C P V L$ & 0.04 & 0.99 & $\mathrm{~N}$ & $D$ & 0.142 & 26.6 & 0.47 \\
\hline 7 & $94,057,039$ & exonic & COLIA2 & 0.10 & 0.98 & $\mathrm{D}$ & $N$ & 0.082 & 26.2 & 0.44 \\
\hline 7 & $1.01 E+08$ & exonic & MUC17 & 0.02 & 0.61 & . & $N$ & 0.003 & 5.6 & 0.04 \\
\hline 7 & $1.51 E+08$ & exonic & CHPF2 & 0.03 & 0.89 & $\mathrm{D}$ & $D$ & 0.049 & 23.3 & 0.32 \\
\hline 8 & $90,936,937$ & exonic & OSGIN2 & 0.38 & 0.03 & $D$ & $D$ & 0.01 & 11.3 & 0.07 \\
\hline 17 & $3,030,476$ & exonic & OR1G1 & 0.05 & 0.01 & & $N$ & 0 & 13.6 & 0.03 \\
\hline 17 & $4,619,845$ & exonic & ARRB2 & 0.04 & 0.90 & $\mathrm{D}$ & $D$ & 0.034 & 32.0 & 0.15 \\
\hline 17 & $6,683,525$ & exonic & FBXO39 & 0.14 & 0.09 & $\mathrm{D}$ & $D$ & 0.024 & 19.8 & 0.17 \\
\hline 17 & $7,733,695$ & exonic & DNAH2 & 0.01 & 0.89 & $N$ & N & 0.004 & 23.8 & 0.11 \\
\hline
\end{tabular}

and organizations of extracellular matrix. Previous study demonstrated ITGA2-deficient mice overexpressed transforming the growth factor TGF $\beta$ [16, 19], which was known to be highly associated with aortic aneurysm and IA. Moreover, both ITGA2 and TTN were involved in hemostasis [3, 30]. Notably, Notch signaling plays a pivotal role during vascular development $[4,18]$. Mutations in NOTCH3 have been identified as the underlying cause of cerebral autosomal dominant arteriopathy with subcortical infarcts and leukoencephalopathy (CADASIL), the most common inherited stroke and dementia syndrome in the group of degenerative small vessel diseases [23]. Our findings demonstrated the therapeutic potential of modifying these signaling in SAHs.

Table 7 The candidate genes in two pedigrees

\begin{tabular}{ll}
\hline Candidate genes in pedigree 1 & Candidate genes in pedigree 2 \\
\hline GPATCH2 & IQGAP3 \\
CFAP65 & DUSP12 \\
PAX3 & ABCB5 \\
GAK & CPVL \\
KIAA0232 & COL1A2 \\
CTTNBP2 & CHPF2 \\
CPA4 & ARRB2 \\
ODF2 & \\
NEURL4 & \\
LLGL2 & \\
BMP7 & \\
LMF2 & \\
\hline
\end{tabular}

\section{Discussion}

Subarachnoid hemorrhage (SAH) is the rarest but most fatal type of stroke, identification of genetic variants that confer susceptibility to SAH is clinically important to prevent it [20]. In the present study, we performed WES for SAH cases and controls, to identify causal variations that associated with SAH risk in China, which enabled us to systemically evaluate protein-altering variants and candidate functional genes.

Across GWAS with a total of 188 samples, we found a genome-wide significant association of SNPs in TPO and PALD1 with SAH risk. These two genes are involved in disorders of thyroid hormonogenesis and formation of

Table 8 The overlapped genes among the results from burden test, SKAT-O analysis and PPI analysis

\begin{tabular}{ll}
\hline Candidate pathogenic genes & \\
\hline MYH1 & CD36 \\
TJP1 & COL14A1 \\
FGFR2 & FGFR4 \\
NCAM1 & FLT4 \\
NOX4 & FZR1 \\
RPS6KA1 & ID4 \\
LAMB2 & IL12RB1 \\
LAMB1 & ITGA2 \\
COL2OA1 & MAPK8 \\
FBLN2 & NCAN \\
POLE & P4HA2 \\
NOTCH3 & RUNX2 \\
ADCY8 & \\
\hline
\end{tabular}


vascular endothelium, respectively. Previous studies of IA have identified SERPINA3 (rs4934) as associated risk loci in the Finnish population, and CSPG2 (rs251124) and HSPG2 (rs3767137) loci as susceptibility sites in the Dutch population. However, in our cohorts, there was no significantly associated signal in these genes, which may due to the different genetic background among the populations.

We then investigated the role of low-frequency variants of intermediate effect in SAH risk through rare SNPs analysis. The pathogenic genes with rare, damaging SNPs were enriched in some pathways related to cellular organization, i.e., degradation of the extracellular matrix; and transcription factor signal, i.e., TGF-beta signaling pathway. TGF-beta signaling plays a vital role in vasculogenesis and maintenance of blood vessel, and is involved in aortic aneurysm and IA. These results highlight the functional importance of rare variations in SAH risk.

The two pedigree samples were mainly used to performed Mendelian inheritance analysis, and it revealed autosomal dominant inheritance of pathogenic genes. In the same time, some potential disease causing variants were also found, such as the gene COL1A2 which was reported to be associated with SAH phenotype [15]. Combing the results from the network analysis of known SAH-associated genes, we obtained a list of candidate susceptibility genes. Among these genes, several were demonstrated to be associated with maintenance of blood vessel, including FBLN2, ITGA2, BMP7, and NOTCH3. NOTCH3 is known to be associated with the most common inherited stroke, CADASIL. These potential targets needed to be further validated in experiment models both in vivo and in vitro, which may facilitate to develop clinical strategies for early detection and intervention.

In conclusion, we have identified a key role for rare variations in SAH and discovered SNPs in new complex loci. However, there are still some limitations to our current study due to the small sample size and availability of family genetic data. In future, the identified candidate genes, i.e., TPO, PALD1 and ITGA2, will be necessary to validate in independent study populations or a larger sample size for Chinese population. Determination of genotypes for SNPs in these genes will guide the development of therapeutic strategies for SAH.

\section{Supplementary information}

Supplementary information accompanies this paper at https://doi.org/10 1186/s13041-020-00620-6.

Additional file 1: Table S1. Sample background information. Table S2. Basic information of sequencing. Table $\mathbf{S 3}$. reads mapping statistics. Table S4. Variation distribution statistics. Table S5. Variation filtering threshold. Table S6. Known genes in SAH. Table S7. Interacted genes with known genes in SAH

\section{Acknowledgements}

We thank the scientific, data management and statistical teams associated with the study.
Authors' contributions

Not Applicable. The author(s) read and approved the final manuscript.

Funding

This study was financed by the National Natural Science Foundation of China (Project No: 81460189).

Availability of data and materials

All data and materials mentioned in this article are available. The original data have been submitted to the public data depository CNGB Nucleotide Sequence Archive (CNSA;https://db.cngb.org/cnsa/) of the China National GeneBank DataBase (CNGBdb) with accession number CNP0000954.

Ethics approval and consent to participate

This study were approved and authorised by the Theoretical committee of baotou central hospital. Ethical review approval number: IRB00001052-11085.

\section{Consent for publication}

The authors declare that there are Consent for publication.

\section{Competing interests}

The authors declare that there are no conflict of commercial interest related to this paper.

Received: 30 December 2019 Accepted: 11 May 2020

Published online: 25 May 2020

\section{References}

1. Adzhubei I, Jordan DM, Sunyaev SR. Predicting functional effect of human missense mutations using PolyPhen-2. Curr Protoc Hum Genet, Chapter 7, Unit7 20; 2013.

2. Anderson CS, Feigin V, Bennett D, Lin RB, Hankey G, Jamrozik K, G. Australasian Cooperative Research on Subarachnoid Hemorrhage Study (2004) Active and passive smoking and the risk of subarachnoid hemorrhage: an international population-based case-control study. Stroke. 2004;35:633-7.

3. Anton Al, Gonzalez-Conejero R, Roldan V, Quiroga T, Sanchez-Vega B, Corral J, Vicente $V$, Mezzano D. Study of 18 functional hemostatic polymorphisms in mucocutaneous bleeding disorders. Ann Hematol. 2010;89:1147-54.

4. Baron-Menguy C, Domenga-Denier V, Ghezali L, Faraci FM, Joutel A. Increased Notch3 activity mediates pathological changes in structure of cerebral arteries. Hypertension. 2017;69:60-70

5. Bolger AM, Lohse M, Usadel B. Trimmomatic: a flexible trimmer for Illumina sequence data. Bioinformatics, btu170. 2014:30(15):2114-20.

6. Bromberg JE, Rinkel GJ, Algra A, van Duyn CM, Greebe P, Ramos LM, van Gijn J. Familial subarachnoid hemorrhage: distinctive features and patterns of inheritance. Ann Neurol. 1995;38:929-34.

7. Browning $B L$, Zhou Y, Browning SR. A one-penny imputed genome from next-generation reference panels. Am J Hum Genet. 2018;103:338-48.

8. Chapman SL, Sicot FX, Davis EC, Huang J, Sasaki T, Chu ML, Yanagisawa H. Fibulin-2 and fibulin-5 cooperatively function to form the internal elastic lamina and protect from vascular injury. Arterioscler Thromb Vasc Biol. 2010; 30:68-74.

9. Chou J, Harvey BK, Chang CF, Shen H, Morales M, Wang Y. Neuroregenerative effects of BMP7 after stroke in rats. J Neurol Sci. 2006; 240:21-9.

10. Chun S, Fay JC. Identification of deleterious mutations within three human genomes. Genome Res. 2009;19:1553-61.

11. De Braekeleer M, Perusse L, Cantin L, Bouchard JM, Mathieu J. A study of inbreeding and kinship in intracranial aneurysms in the Saguenay lac-saintJean region (Quebec, Canada). Ann Hum Genet. 1996;60:99-104.

12. de Rooij NK, Linn FH, van der Plas JA, Algra A, Rinkel GJ. Incidence of subarachnoid haemorrhage: a systematic review with emphasis on region, age, gender and time trends. J Neurol Neurosurg Psychiatry. 2007;78:1365-72.

13. Do R, Kathiresan S, Abecasis GR. Exome sequencing and complex disease: practical aspects of rare variant association studies. Hum Mol Genet. 2012; 21:R1-9.

14. Feigin VL, Rinkel GJ, Lawes CM, Algra A, Bennett DA, van Gijn J, Anderson CS. Risk factors for subarachnoid hemorrhage: an updated systematic review of epidemiological studies. Stroke. 2005;36:2773-80. 
15. Gan Q, Liu Q, Hu X, You C. Collagen type I alpha 2 (COL1A2) polymorphism contributes to intracranial aneurysm susceptibility: a meta-analysis. Med Sci Monit. 2017;23:3240-6.

16. Girgert R, Martin M, Kruegel J, Miosge N, Temme J, Eckes B, Muller GA, Gross O. Integrin alpha2-deficient mice provide insights into specific functions of collagen receptors in the kidney. Fibrogenesis Tissue Repair. 2010;3:19.

17. Hackett ML, Anderson CS. Health outcomes 1 year after subarachnoid hemorrhage: an international population-based study. Australian Cooperative Research on Subarachnoid Hemorrhage Study Group. Neurology. 2000;55:658-62.

18. Henshall TL, Keller A, He L, Johansson BR, Wallgard E, Raschperger E, Mae MA, Jin S, Betsholtz C, Lendahl U. Notch3 is necessary for blood vessel integrity in the central nervous system. Arterioscler Thromb Vasc Biol. 2015; 35:409-20.

19. Holtkotter $O$, Nieswandt B, Smyth N, Muller W, Hafner M, Schulte V, Krieg T, Eckes B. Integrin alpha 2-deficient mice develop normally, are fertile, but display partially defective platelet interaction with collagen. J Biol Chem. 2002;277:10789-94.

20. Hop JW, Rinkel GJ, Algra A, van Gijn J. Case-fatality rates and functional outcome after subarachnoid hemorrhage: a systematic review. Stroke. 1997; 28:660-4.

21. Ioannidis NM, Rothstein JH, Pejaver V, Middha S, McDonnell SK, Baheti S, Musolf A, Li Q, Holzinger E, Karyadi D, Cannon-Albright LA, Teerlink CC, Stanford JL, Isaacs WB, Xu J, Cooney KA, Lange EM, Schleutker J, Carpten JD, Powell IJ, Cussenot O, Cancel-Tassin G, Giles GG, Maclnnis RJ, Maier C, Hsieh CL, Wiklund F, Catalona WJ, Foulkes WD, Mandal D, Eeles RA, Kote-Jarai Z, Bustamante CD, Schaid DJ, Hastie T, Ostrander EA, Bailey-Wilson JE, Radivojac P, Thibodeau SN, Whittemore AS, Sieh W. REVEL: an ensemble method for predicting the pathogenicity of rare missense variants. Am J Hum Genet. 2016;99:877-85.

22. Jagadeesh KA, Wenger AM, Berger MJ, Guturu H, Stenson PD, Cooper DN, Bernstein JA, Bejerano G. M-CAP eliminates a majority of variants of uncertain significance in clinical exomes at high sensitivity. Nat Genet. 2016; 48:1581-6.

23. Joutel A, Corpechot C, Ducros A, Vahedi K, Chabriat H, Mouton P, Alamowitch S, Domenga V, Cecillion M, Marechal E, Maciazek J, Vayssiere C, Cruaud C, Cabanis EA, Ruchoux MM, Weissenbach J, Bach JF, Bousser MG, Tournier-Lasserve E. Notch3 mutations in CADASIL, a hereditary adult-onset condition causing stroke and dementia. Nature. 1996;383:707-10.

24. Kissela BM, Sauerbeck L, Woo D, Khoury J, Carrozzella J, Pancioli A, Jauch E, Moomaw CJ, Shukla R, Gebel J, Fontaine R, Broderick J. Subarachnoid hemorrhage: a preventable disease with a heritable component. Stroke. 2002:33:1321-6

25. Lee S, Emond MJ, Bamshad MJ, Barnes KC, Rieder MJ, Nickerson DA, Team NGESP-ELP, Christiani DC, Wurfel MM, Lin X. Optimal unified approach for rare-variant association testing with application to small-sample case-control whole-exome sequencing studies. Am J Hum Genet. 2012;91:224-37.

26. Li H, Durbin R. Fast and accurate long-read alignment with burrowswheeler transform. Bioinformatics. 2010;26:589-95.

27. Mather CA, Mooney SD, Salipante SJ, Scroggins S, Wu D, Pritchard CC, Shirts $\mathrm{BH}$. CADD score has limited clinical validity for the identification of pathogenic variants in noncoding regions in a hereditary cancer panel. Genet Med. 2016;18:1269-75.

28. McKenna A, Hanna M, Banks E, Sivachenko A, Cibulskis K, Kernytsky A, Garimella K, Altshuler D, Gabriel S, Daly M, DePristo MA. The genome analysis toolkit: a MapReduce framework for analyzing next-generation DNA sequencing data. Genome Res. 2010;20:1297-303.

29. Nelson MR, Wegmann D, Ehm MG, Kessner D, Jean PS, Verzilli C, Shen J, Tang Z, Bacanu SA, Fraser D, Warren L, Aponte J, Zawistowski M, Liu X, Zhang H, Zhang Y, Li J, Li Y, Li L, Woollard P, Topp S, Hall MD, Nangle K, Wang J, Abecasis G, Cardon LR, Zollner S, Whittaker JC, Chissoe SL, Novembre J, Mooser V. An abundance of rare functional variants in 202 drug target genes sequenced in 14,002 people. Science. 2012;337:100-4.

30. Norton N, Li D, Rampersaud E, Morales A, Martin ER, Zuchner S, Guo S, Gonzalez M, Hedges DJ, Robertson PD, Krumm N, Nickerson DA, Hershberger RE, L. National Heart, G. O. E. S. P. Blood Institute \& T. the Exome Sequencing Project Family Studies Project. Exome sequencing and genome-wide linkage analysis in 17 families illustrate the complex contribution of TTN truncating variants to dilated cardiomyopathy. Circ Cardiovasc Genet. 2013;6:144-53.
31. Rivolta CM, Esperante SA, Gruneiro-Papendieck L, Chiesa A, Moya CM, Domene S, Varela V, Targovnik HM. Five novel inactivating mutations in the thyroid peroxidase gene responsible for congenital goiter and iodide organification defect. Hum Mutat. 2003;22:259.

32. Schievink WI, Schaid DJ, Michels W, Piepgras DG. Familial aneurysmal subarachnoid hemorrhage: a community-based study. J Neurosurg. 1995;83: 426-9.

33. Schwarz JM, Rodelsperger C, Schuelke M, Seelow D. MutationTaster evaluates disease-causing potential of sequence alterations. Nat Methods. 2010;7:575-6.

34. Stoupa A, Chaabane R, Gueriouz M, Raynaud-Ravni C, Nitschke P, BoleFeysot C, Mnif M, Ammar Keskes L, Hachicha M, Belguith N, Polak M, Carre A. Thyroid hypoplasia in congenital hypothyroidism associated with thyroid peroxidase mutations. Thyroid. 2018;28:941-4.

35. Szklarczyk D, Gable AL, Lyon D, Junge A, Wyder S, Huerta-Cepas J, Simonovic M, Doncheva NT, Morris JH, Bork P, Jensen LJ, Mering CV. STRING v11: protein-protein association networks with increased coverage, supporting functional discovery in genome-wide experimental datasets. Nucleic Acids Res. 2019;47:D607-13.

36. Wallgard E, Nitzsche A, Larsson J, Guo X, Dieterich LC, Dimberg A, Olofsson T, Ponten FC, Makinen T, Kalen M, Hellstrom M. Paladin (X99384) is expressed in the vasculature and shifts from endothelial to vascular smooth muscle cells during mouse development. Dev Dyn. 2012;241:770-86.

37. Wang K, Li M, Hakonarson H. ANNOVAR: functional annotation of genetic variants from high-throughput sequencing data. Nucleic Acids Res. 2010;38: e164.

38. Wang PS, Longstreth WT Jr, Koepsell TD. Subarachnoid hemorrhage and family history. A population-based case-control study. Arch Neurol. 1995;52: 202-4.

39. Yang J, Lee SH, Goddard ME, Visscher PM. GCTA: a tool for genome-wide complex trait analysis. Am J Hum Genet. 2011;88:76-82.

40. Zhang J, Liu G, Arima H, Li Y, Cheng G, Shiue I, Lv L, Wang H, Zhang C, Zhao J, Anderson CS, Investigators C. Incidence and risks of subarachnoid hemorrhage in China. Stroke. 2013;44:2891-3.

\section{Publisher's Note}

Springer Nature remains neutral with regard to jurisdictional claims in published maps and institutional affiliations.
Ready to submit your research? Choose BMC and benefit from:
- fast, convenient online submission
- thorough peer review by experienced researchers in your field
- rapid publication on acceptance
- support for research data, including large and complex data types
- gold Open Access which fosters wider collaboration and increased citations
- maximum visibility for your research: over $100 \mathrm{M}$ website views per year
At BMC, research is always in progress.
Learn more biomedcentral.com/submissions 